\title{
PRINCÍPIOS FAIR E INTEROPERABILIDADE: REPRODUTIBILIDADE COM O USO DE MODELAGEM CONCEITUAL ORIENTADA A ONTOLOGIAS
}

\author{
FAIR PRINCIPLES AND INTEROPERABILITY: \\ REPRODUCIBILITY USING ONTOLOGY-DRIVEN \\ CONCEPTUAL MODELLING
}

\author{
Claúdio José Silva Ribeiroa \\ Luiz Olavo Bonino da Silva Santos ${ }^{b}$ \\ João Luiz Ribeiro Moreirac
}

\begin{abstract}
RESUMO
Introdução: o desenvolvimento de modelos conceituais como artefatos de referência para compreensão de domínios do conhecimento contribui para auxiliar na redução da distância semântica entre a representação e a interpretação das informações. Tomando por base o contexto do compartilhamento de dados nos esforços do acesso aberto à produção em Ciência \& Tecnologia, bem como as dificuldades originadas pela crise da reprodutibilidade, este relato analisa a existência de modelos semânticos para representação no contexto das GLAM (Galleries, Libraries, Archives and Museums). Objetivo: propor a análise desse rol de modelos em busca da homogeneização das representações dos objetos componentes das pesquisas, à luz dos princípios FAIR e apresentar um modelo de referência que permita a convergência nas representações. Metodologia: combinando métodos e abordagens epistemológicas, este position paper usou pesquisa exploratória com análise bibliográfica e documental para identificar padrões de representação. Resultados: apresenta o conjunto de instrumentos identificados e categorizados segundo sua aplicabilidade, além de uma proposta de modelo semântico. Conclusões: este relato aponta para a necessidade de convergência de padrões de representação em conjunto com modelos conceituais, como forma de viabilizar a interoperabilidade e o uso dos princípios FAIR.
\end{abstract}

\footnotetext{
a Doutor em Ciência da Informação pela Universidade Federal Fluminense (UFF/MCT-IBICT). Professor da Universidade Federal do Estado do Rio de Janeiro (UNIRIO). E-mail: claudio.ribeiro@unirio.br.

b PhD, Associate Professor, Services and Cyber-Security, Electrical Engineering, Mathematics and Computer Science - University of Twente - Netherlands - E-mail: l.o.boninodasilvasantos@utwente.nl.

c PhD, Assistant Professor, Services and Cyber-Security, Electrical Engineering, Mathematics and Computer Science - University of Twente - Netherlands - E-mail:

j.luizrebelomoreira@utwente.nl.
}

Inf. Inf., Londrina, v. 25, n. 3, p. $28-51$, jul./set. 2020. http://www.uel.br/revistas/informacao/ 
Descritores: Reprodutibilidade. FAIR. Interoperabilidade. Modelo Conceitual. Representação.

\section{INTRODUÇÃO}

Com o incremento do interesse no compartilhamento de dados e informação entre pesquisadores, pode-se inferir que os caminhos para o desenvolvimento de investigações estão sendo impulsionados na direção da Ciência Aberta. Projetos de pesquisas transnacionais, como por exemplo os desenvolvidos no contexto da pandemia do Coronavírus1, promoveram esses deslocamentos e vêm ocasionando reflexos não apenas no percurso metodológico adotado, mas também na dinâmica de representação documental.

Por outro lado, as dificuldades apontadas em trabalhos correlatos sobre reúso dos objetos resultantes das investigações culminaram em percepções que apontam na direção dos problemas na reprodução de pesquisas (IOANNIDIS, 2005; NOSEK; SPIES; MOTYL, 2012). Baker (2016) convalida essa abordagem quando revela em sua avaliação: $52 \%$ dos pesquisadores entrevistados concordaram com a argumentação de que existe uma crise de reprodutibilidade. Baker continua e apresenta outros resultados que podem traduzir bem a dificuldade na representação dos objetos de pesquisa. Segundo esse autor, $70 \%$ dos pesquisadores entrevistados falharam ao tentar reproduzir o experimento de outros cientistas. Um resultado surpreendente foi que cerca de $50 \%$ dos entrevistados também relataram falha em tentar reproduzir o seu próprio experimento.

A visão da representação de obras documentais remete ao papel do profissional da informação. Nesse sentido, ao realizar um sobrevoo por momentos históricos vivenciados pelos profissionais de Library and Information Science, é possível asseverar que os bibliotecários exercem o papel de

\footnotetext{
1 Iniciativa VODAN (Virus Outbreak Data Network), com o intuito de tornar os dados do vírus SARS CoV-2 alinhados com os princípios FAIR. O objetivo final é que esses dados sejam Findable, Accessible, Interoperable e Reusable para impulsionar as pesquisas na pandemia da COVID-19. Disponível em: https://www.go-fair.org/implementation-networks/overview/vodan/. Acesso em: 1ㅇ ago. 2020.
} 
guardiões do conhecimento e para desempenhar essa função precisaram desenvolver habilidades para representá-lo. Para tanto, o estudo e uso de metateorias foi elemento de destaque na condução dessas representações (ZAHER; GOMES, 1972; ROBREDO, 2003). Cabe lembrar que o prefixo meta pode levar para uma interpretação sobre, ou ainda, uma representação mais abstrata - como por exemplo um apontador ou referência (JAPIASSÚ; MARCONDES, 2006).

Assim, moldou-se o entendimento sobre metadados bibliográficos como elementos que permitem o registro de obras nas bibliotecas, por meio da descrição de suas principais características. A representação de metadados pode ser descritiva (metadados que descrevem um objeto), mas também pode ser temática (uso de vocabulários e termos para auxiliar na descrição de contexto para preenchimento dos metadados). A junção dessas possíveis representações com o incremento dos vocabulários presentes em diferentes campos do conhecimento leva para além dos processos convencionais de representação.

Existem diferentes padrões para registro de metadados no contexto das GLAM (Galleries, Libraries, Archives and Museums) e estão sendo adotados outros tipos de modelos semânticos para representação. $O$ cenário que se apresenta é de evolução no tratamento da informação, na medida em que vem provocando alterações até mesmo no perfil das equipes que trabalham com produção de conhecimento em Ciência \& Tecnologia, especialmente em processos de descrição para representação e organização dos objetos componentes das investigações.

A existência de vários objetos com tipologia diversa pode indicar a necessidade de ir além nos processos convencionais de tratamento da informação. Ao revisitar algumas percepções sobre a exploração de literatura técnica, Latour (2000) introduzia uma visão sobre esse assunto tão relevante no contexto da Ciência na atualidade:

[...] Para o leigo, o estudo da ciência e da tecnologia significaria então analisar o discurso dos cientistas, contar citações, fazer cálculos bibliométricos ou realizar estudos semióticos dos textos científicos e de sua etnografia, ou seja, usar técnicas de crítica literária na literatura técnica. Por mais interessantes e necessários que esses estudos sejam, não são suficientes se quisermos 
acompanhar o trabalho de cientistas e engenheiros; afinal eles não rascunham, não escrevem e leem artigos científicos 24 horas por dia. Cientistas e Engenheiros sempre alegam que por trás dos textos técnicos há algo muito mais importante do que qualquer coisa que eles escrevam (LATOUR, 2000, p. 105).

Adicionalmente, a adoção dos princípios FAIR (acrônimo para Findable, Accessible, Interoperable e Reusable) discute a melhoria no reúso de dados de pesquisa, propondo de forma destacada "a necessidade desses objetos [de pesquisa] manterem uma contextualização precisa para transmitirem informação e conhecimento ao longo do tempo" (HENNING; RIBEIRO; SALES; MOREIRA; SANTOS, 2018, p. 5048).

Essa contextualização, em essência, pode ser traduzida por representações formais para expressar o conhecimento. Foi nessa direção que, com a ótica do compartilhamento, surgiu a intenção de buscar apoio na atividade de modelagem conceitual como forma de reduzir a distância semântica entre os atos de representar e interpretar ativos de informação² (GUIZZARDI, 2005).

Contudo, vale destacar que a modelagem conceitual é tarefa que pode produzir, na área da Ciência da Informação, diferentes artefatos, desde um esboço gráfico sobre conceitos em uso, até representar um modelo bibliográfico, ou ainda apresentar um conjunto de estruturas conceituais (taxonomias, tesauros e esquemas de representação).

A proposta deste relato é apresentar a homogeneização na realização dessa tarefa e promover o seu possível engendramento no processo de pesquisa. Essa concertação tem como ponto central o uso da modelagem conceitual como modo de viabilizar o incremento da interoperabilidade semântica com base nos princípios FAIR.

2 Para abarcar esses diversos objetos portadores de informação em projetos de pesquisa, utilizou-se o termo ativo de informação, enunciado em Ribeiro (2014). Propõe-se assim que esse termo seja abrangente, caracterizando a união de objetos de pesquisa, sejam publicações científicas ou insumos coletados e utilizados ao longo da pesquisa (dados de pesquisa), e promovendo o incremento no objeto de estudos da Ciência da Informação. Wilkinson, Sansone, Schultes et al. (2018) convalidam essa proposta quando observam que é importante aplicar os princípios FAIR não apenas aos dados, mas a todos os objetos digitais de pesquisa, garantindo que todos os componentes do processo de pesquisa estejam disponíveis de forma transparente e com a possibilidade de serem reprodutíveis. 
Este position paper $^{3}$ partiu de pesquisa exploratória com análise bibliográfica e documental para identificar os padrões de representação (LAKATOS; MARCONI, 2017). Fazendo uso da Filosofia Analítica (RIBEIRO; PEREIRA, 2013) em conjunto com a análise de conteúdo (MENDONÇA, 2007), foi desenvolvida uma revisão sistemática não estruturada da literatura. Isso permitiu a identificação de modelos semânticos, nas dimensões de sintaxe (verbal), semântica (contexto e expressão) e pragmática (uso).

Vale ressaltar que, apesar de este relato fazer uso da modelagem conceitual orientada a ontologias, não foi objetivo da pesquisa pormenorizar os princípios e requisitos necessários ao processo de concepção do modelo. $O$ aporte teórico adotado neste relato buscou trabalhar com especificações que serão implementadas por meio da modelagem conceitual, além dos formalismos (vocabulários e, possivelmente, ontologias) como instrumentos que possibilitam a descrição de ligações semânticas entre ativos de informação, sejam dados de pesquisa ou publicações científicas.

Além desta introdução que está sendo finalizada aqui, este relato conta com mais três seções. A próxima irá tratar dos pilares ligados à busca da reprodutibilidade; em seguida, serão apresentados os resultados e, por fim, a última seção será dedicada às considerações finais.

\section{EM BUSCA DA REPRODUTIBILIDADE: DA REPRESENTAÇÃO AO USO DE MODELAGEM CONCEITUAL}

A possibilidade de reutilizar ativos de informação originários de projetos de pesquisa está intrinsecamente ligada a dois aspectos que podem ser considerados pilares de sustentação para a reprodutibilidade: a capacidade de representação da informação e o processo de interoperabilidade entre domínios.

Quando, por conta deste estudo, adentrou-se no terreno do reúso de dados e informação em projetos de investigação houve o encontro com um assunto que

\footnotetext{
${ }^{3}$ A classificação de position paper é encontrada na literatura de metodologia científica quando se tem o objetivo de discutir aspectos e assuntos inovadores, além de pressupor o alcance de novos resultados (WIKIPEDIA, 2020). Marlow (2006) e Zins, Debons, Beghtol, et al. (2007) utilizaram este tipo de produção no contexto da Ciência da Informação.
} 
tem norteado as discussões em diferentes áreas do conhecimento: o uso dos princípios FAIR para viabilizar esse compartilhamento. Com requisitos que recomendam a utilização de vocabulários e modelos semânticos, o uso desses princípios pode nortear o processo de representação e contribuir para tornar os ativos interoperáveis (HENNING; RIBEIRO; SALES; MOREIRA; SANTOS, 2018).

Como resultado do processo de representação, podem surgir diferenças na interpretação dos objetos representados. $O$ artefato derivado da atividade de modelagem permite a elaboração de instrumento de referência para mediar essas diferenças (GUIZZARDI, 2007).

\subsection{A NoçÃo de REPROdUtibILIDADE}

Quando estamos tratando do incremento da produção em Ciência Aberta, a reprodutibilidade pode ser considerada um marco para o sucesso na evolução das pesquisas (SANDVE; NEKRUTENKO; TAYLOR; HOVIG, 2013). Esses mesmos autores justificam esta afirmação quando observam: "We want to emphasize that reproducibility is not only a moral responsibility with respect to the scientific field, but that a lack of reproducibility can also be a burden for you as an individual researcher[...]'.

Segundo a National Academy of Sciences, Engineering and Medicine (2019), a noção de reprodutibilidade tem sido debatida vários campos de estudo e com diversas interpretações, muitas vezes contraditórias. Termos como "Replicação" e "Repetição" podem ser encontrados em diferentes áreas do conhecimento. Todavia, muitas das publicações sobre reprodutibilidade têm centrado as discussões na ausência de códigos e metadados que descrevam em detalhes os métodos e resultados obtidos.

Adotou-se neste relato a proposta da National Academy of Sciences, que nos lembra: "[...] reproducibility is obtaining consistent results using the same input data; computational steps; methods, and code; and conditions of analisys. This definition is synonymous with 'computational reproducibility', [...]' (NATIONAL ACADEMY OF SCIENCES, ENGINEERING, AND MEDICINE, 2019, online). Stodden (2015) já apontava um entendimento semelhante quando 
afirmava que a reprodutibilidade pode ser entendida como "[...] the same results can be obtained from the data and code used in the original study".

No contexto das Ciências Sociais, Barba (2018) constata que não existem diferenças na compreensão da reprodutibilidade, pois o tratamento dado nessa área do conhecimento aponta a convergência entre o uso das definições de replicações e repetições de investigações. Em síntese, pode-se inferir que no contexto das GLAM é possível adotar essas definições como sinônimos para reprodutibilidade.

Portanto, tendo em vista o diagnóstico evidenciado em Baker (2016) sobre a existência da crise de reprodutibilidade, e em função do cenário apresentado, é possível afirmar que o caminho para alcançar a reprodutibilidade deve estar suportado por artefatos que façam a mediação entre a representação e a interpretação dos ativos de informação.

\subsection{Princípios FAIR e os Reflexos na RepresentaçÃo}

Partindo-se das visões apresentadas em Henning, Ribeiro, Sales, Moreira e Santos (2018) e Henning, Ribeiro, Santos e Santos (2019), propõe-se que estes princípios também possam nortear a evolução dos processos de interoperabilidade e reúso não apenas para dados mas também para o conteúdo de publicações científicas, denominados neste relato de ativos de informação, adotando-se:

- Ativos de informação interoperáveis: prontos para serem combinados (e intercambiados) com outros ativos, com mediação de pessoas ou de scripts automatizados em computadores;

- Ativos de informação reutilizáveis: prontos para serem usados em pesquisas futuras e para serem posteriormente processados com 0 uso de métodos computacionais.

Para satisfazer a esses princípios é possível estender e destacar os requisitos, tanto para metadados quanto para os outros tipos de ativos de informação descritos por eles (metadados), que devem:

- possuir identificadores persistentes e universais; 
- ser representados por meio de uma linguagem formal, acessível, compartilhada e amplamente aplicável para a representação do conhecimento - ontologias de domínio, modelos semânticos de dados, modelos semânticos de metadados, além de vocabulários e tesauros que seguem os princípios FAIR;

- incluir referências qualificadas para outros metadados, dados ${ }^{4} \mathrm{e}$ publicações. Uma "referência qualificada" descreve a sua intenção. As relações entre ativos de informação ou entre elementos desses ativos precisam ser descritas semanticamente.

- a proveniência de todos os elementos precisa ser devidamente informada. É preciso conhecer se estes foram reutilizados, com detalhes sobre a descrição, geração, coleta, histórico de disponibilização e reúso, além de indicar se houve processo de transformação para o caso de serem derivados de alguma operação. Esse fluxo de informação deve ser descrito em um formato legível por mecanismos automatizados;

- ter licenças claras e acessíveis. Esse requisito se refere à noção de interoperabilidade legal ${ }^{5}$. Os direitos poderão limitar severamente a reutilização dos elementos envolvidos;

- seguir o uso de padrões compartilhados em comunidades de domínio.

É possível notar que esses requisitos destacam a importância da representação com o uso de vocabulários e modelos semânticos; nesse sentido, Guizzardi (2019) fornece o suporte para afirmar-se que a capacidade de representação dos ativos pode conduzir para o campo da modelagem conceitual, pois:

[...] we should be reminded that interoperability is not about finding ways to connect data artifacts but ultimately about

\footnotetext{
${ }^{4}$ Quando os princípios FAIR se referem a dados, dizem respeito a qualquer tipo de objeto digital. A distinção nos princípios entre dados e metadados concerne à distinção entre objetos digitais e um tipo específico de ativo de informação que descreve cada um desses objetos digitais, e.g., dados (no sentido clássico) e seus metadados, ontologia e seus metadados, software e seus metadados etc.

${ }^{5}$ Não há intenção de apresentar neste relato a interoperabilidade legal e todas as nuances envolvidas no respectivo licenciamento.
} 
affording the interoperation of humans mediated by these artifacts. Information artifacts are instruments used by humans to harmonize their conceptualizations and, hence, interoperability approaches succeed to the extent that they can safely connect these conceptualizations.

Tendo essa ótica como pano de fundo, é preciso explorar a interoperabilidade na medida em que pode ser considerada como um dos aspectos centrais na implementação de FAIR (WILKINSON; DUMONTIER; AALLBERSBERG et al., 2016).

\subsection{INTEROPERABILIDADE SEMÂNTICA}

Heiler (1995) introduzia a discussão sobre a interoperabilidade semântica para além dos aparatos tecnológicos, destacando em especial a relação centrada na capacidade de comunicação entre emissores e receptores, desde o uso de soluções de TI até a dinâmica de comunicação interpessoal, pois "interpretation of shared data in an unambiguously way, ensuring that the understanding of the information is the same for senders and receivers". Nesse sentido, pode-se afirmar que a interoperabilidade é uma engrenagem essencial que torna transparente para os usuários não só a convivência entre diferentes ambientes tecnológicos, mas também entre métodos de trabalho e procedimentos.

Os princípios FAIR assumem um ambiente heterogêneo e distribuído, em que dados, serviços e computadores interagem tão autonomamente quanto possível. Para que esse grau crescente de autonomia seja atingido, é necessário que esses participantes possam interagir e interoperar em diversos níveis. Historicamente, a computação superou diversos desafios de interoperabilidade. Da impossibilidade de compartilhamento de dados e programas nos computadores iniciais, por eles os únicos com controles, configurações e interfaces próprias, passou-se à possibilidade crescente de interoperabilidade quando foram superados os desafios relacionados a sistemas operacionais, infraestruturas de rede, protocolos de comunicações, formatos de representação de dados e modelos de controle.

Com os avanços tecnológicos atuais é possível assumir que uma mensagem enviada por um sistema computacional seja recebida por outro 
sistema. Isso ocorre independentemente das redes às quais esses sistemas estejam conectados ou do formato de serialização da mensagem e sua estrutura e sintaxe. Tecnologias presentes na internet, como TCP/IP, o serviço World Wide Web (www), o protocolo HTTP, a linguagem XML e a arquitetura RDF, endereçam algumas dessas questões. Portanto, o próximo grande desafio é a interoperabilidade semântica.

Em termos informais, um dos objetivos dos princípios FAIR é que "as máquinas entendam o que queremos dizer". Obviamente, para que isso aconteça, primeiramente nós temos de saber o que queremos dizer. Como consequência, precisamos de diferentes tipos de descrições semânticas que sejam apropriadas para diferentes objetivos. Por exemplo, tornar claro e formalizar o acordo das intenções semânticas entre agentes humanos, descrições formais para processamento por máquinas etc.

Na visão de Guizzardi (2019), a interoperabilidade [semântica] é utilizada para solucionar os problemas advindos da necessidade de combinar diferentes espaços de informação, provendo informação de maneira unificada. Nesse sentido, percebe-se que as dificuldades e obstáculos encontrados para convergir os ambientes tecnológicos e padronizar métodos de trabalho tornam complexa a utilização e reutilização dos dados, além de indicar a necessidade de criação de uma infraestrutura global de compartilhamento.

Essa infraestrutura possibilitaria a interoperabilidade dos dados, viabilizando a semântica consensual e usando padrões e protocolos já aprovados (HENNING; RIBEIRO; SANTOS; SANTOS, 2019).

Ross-Hellauer, Fecher, Sheares e Rodrigues (2019) convalidam essa intenção sobre a infraestrutura quando apresentam uma proposta de arquitetura conceitual apoiada em padrões de interoperabilidade para repositórios e camadas de disseminação de conteúdo, para dimensões: sistema, estrutural, sintática e semântica. Portanto, essa jornada culmina com o uso de instrumentos mediadores que devem permear as diferentes camadas de representação. 


\subsection{Modelagem Conceitual Como Mediador da INTEROPERABiLIDADE SEMÂNTICA}

A atividade de modelagem permite $\mathrm{o}$ esboço e a análise do comportamento dos objetos antes da implementação. Modelos podem representar substratos da realidade em ambiente controlado, podendo responder a indagações e permitir simulações. Keneth Craik destacava o uso de "small-scale model" como alternativa válida para representação da realidade (CRAIK, 1952 apud SOWA, 2000, p. 364).

Ao abordar aspectos ligados à representação de domínios, Mylopoulos já apresentava de maneira objetiva a utilização de modelagem conceitual como atividade fundamental para compreendermos o mundo:

Conceptual modelling, on the other hand, has been concerned with life-size models of portions of the [physical and social] world to be made available to human users, for purposes of understanding and communication. [...] The adequacy of a conceptual modelling notation rests on its contribution to the construction of models of reality that promote a common understanding of that reality among their human users (MYLOPOULOS, 1992, p. 3).

Kent (2000) assevera que modelos são abordagens simples e estruturadas para representar dados e informação, além de viabilizar a implementação de sistemas de informação por meio da utilização da tecnologia dos computadores. Japiassú e Marcondes (2006, p. 190) registram que modelo é um "[...] Objeto que serve de parâmetro para construção ou criação de outros. Qualquer coisa ou pessoa que se toma como inspiração ou ideal a ser imitado ou copiado."

Allemang e Hendler (2011, p. 17) afirmam que "models are used to organize human thought in the form of explanations. When we understand how a phenomenon results from other basics principles, we gain a number of advantages".

Fenlon (2019) afirma que modelos e objetos de pesquisa nas Digital Humanities Collections possuem uma forte relação, pois podem explicitar como relações são declaradas e instrumentalizadas por padrões (e formalismos) disponíveis. Moreira (2019) destaca a importância das relações conceituais e a sua materialização em modelos como elementos essenciais no uso de Sistemas de 
Organização do Conhecimento (SOC). Em recente comunicação na 38a Conferência sobre Modelagem Conceitual (ER2019), realizada em Salvador (BA), Weber (2019) apontou para a importância dos modelos conceituais na comunicação e documentação, como forma de obter melhoria nos processos de desenvolvimento para sistemas de informação que envolvem múltiplos tipos de dados.

Como comentado na introdução deste relato, é preciso convalidar aqui que a designação "modelagem conceitual" para a área de Ciência da Informação pode assumir vários significados. É possível destacar:

- elaboração de representação gráfica sobre conceitos e suas relações (INAZAWA; BAPTISTA, 2012; MORAES; SIMÕES, 2017);

- representação em modelos conceituais bibliográficos (MORENO, 2009; PADRON; CRUZ; SILVA, 2018; CERRÃO; CASTRO, 2019);

- desenvolvimento de diagrama Entidade e Relacionamento (E-R) usado para projeto de base de dados (GUIMARÃES; ROGGE, 2019);

- referência à análise de modelos arquiteturais existentes, como por exemplo o OAIS (FLORES; PRADEBON; CÉ, 2017; SILVA; SIEBRA; ALBUQUERQUE, 2018);

- arcabouço teórico-metodológico que pode sustentar a investigação de uma temática (PAIVA, 2012; JAPPUR; FORCELLINI; SPANHOL, 2014; CARAN; BIOLCHINI, 2016; SIMIONATO; SANTOS, 2017; ALMEIDA; SILVA, 2019).

A noção de modelagem conceitual tratada neste relato segue os pressupostos de explicação e explicitação para formalização de conceitos e associações. Esses pressupostos foram reunidos em Verdonck, Gailly, Pergl, Guizzardi, Martins e Pastor (2019) e Almeida, Falbo e Guizzardi (2019). A noção também é corroborada em Machado, Almeida e Souza (2020), quando indicam a aproximação de modelagem conceitual da temática de Sistemas de Informação, na área de Knowledge Organization. 


\subsubsection{Uso de Modelagem Conceitual Orientada a Ontologias (Ontology- Driven Conceptual Model)}

O atendimento à variada tipologia de requisitos leva esta discussão para o terreno da modelagem semântica, pois, como observa Croft (2003, p. 133), podem existir múltiplas representações utilizando diferentes níveis de abstração. Isso pode convalidar a intenção proposta por este relato em produzir um "semantic map model".

As discussões encaminhadas no campo dos mapas semânticos podem ser atualizadas para o contexto da modelagem conceitual para representação do conhecimento, pois "Conceptual models were introduced to increase understanding and communication of a system or domain among stakeholders". (VERDONCK; GAILLY; PERGL; GUIZZARDI; MARTINS; PASTOR, 2019, p. 92).

Verdonck, Gailly, Pergl, Guizzardi, Martins e Pastor continuam e afirmam que investigar os processos de representação do conhecimento na modelagem conceitual é tarefa essencial, pois permitirá explicitar e explicar o conteúdo, além de possibilitar expressar a articulação entre os elementos do domínio analisado. Guizzardi (2020) convalida essa afirmação quando registra a necessidade de elucidar relações entre referentes em um domínio, pois "we need more than merely terminological resources, but formal, shared and explicit representations of conceptualizations, or, what the area of knowledge representation has conventionally called ontologies ${ }^{0 \prime}$ (GUIZZARDI, 2020, p. 186, grifo do autor).

Para representar a modelagem conceitual semântica, foi utilizado um conjunto de estereótipos, a partir de categorias, hierarquias e relações (CROFT, 2003) (Figura 1). Para categorias e hierarquias, foram trabalhados: Kind, Subkind, Phase, Relator e Mode. Para relações, foram utilizados: Formal, Material, Mediation, Characterization e ComponentOf (GUIZZARDI, 2005; 2007; ALMEIDA; FALBO; GUIZZARDI, 2019).

Portanto, pode-se inferir que modelagem conceitual, normas, metadados e formalismos compõem um contexto importante na sustentação da

\footnotetext{
${ }^{6}$ Guizzardi trata a discussão das ontologias no contexto da elaboração de artefatos originários do uso de modelagem conceitual.
} 
interoperabilidade semântica tão desejada entre repositórios e serviços de armazenamento.

\section{A MODELAGEM CONCEITUAL GERANDO ARTEFATO MEDIADOR PARA A CONVERGÊNCIA DE REPRESENTAÇÕES}

O domínio escolhido para o desenvolvimento desta investigação foi o das instituições abrangidas pelas GLAM. Pode-se afirmar que nos últimos anos houve incremento de estudos para representação e compartilhamento em projetos nessas instituições. Todavia, existem múltiplos catálogos que podem ser considerados como forma de mediação, os quais dependem de alguma colaboração para convergirem (SIMIONATO; ARAKAKI; SANTOS, 2018).

Em revisão de literatura não exaustiva sobre o domínio, foram identificados diferentes modelos semânticos para descrição de ativos de informação. A busca de convergência poderá ser obtida com a (re)utilização de itens identificados neste trabalho, usados em esforços ligados à representação descritiva de ativos, mas também contribuirá para o enriquecimento das representações. O Quadro 1 apresenta um rol de modelos e a proposta de categorização para análise (cobertura). É possível notar que um determinado modelo pode atender a mais de um escopo.

Quadro 1- Esforços para padronização semântica no domínio das GLAM.

\begin{tabular}{|c|c|c|}
\hline Formalismo/Modelo Semântico & Escopo & Cobertura \\
\hline 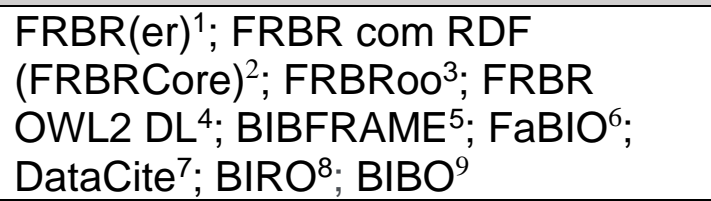 & $\begin{array}{l}\text { Registros } \\
\text { Bibliográficos }\end{array}$ & \\
\hline $\begin{array}{l}\text { METS }^{10} ; \text { MODS }^{11} ; \text { TextMD }^{12} ; \text { EAD }^{13} ; \\
\text { VRACore }^{14} ; \text { LIDO }^{15} ; \text { MIDAS-Heritage, } \\
\text { CARARE Metadata Schema, } \\
\text { CDWA }^{16} ; \text { DCMI }^{17}\end{array}$ & $\begin{array}{l}\text { Registro para } \\
\text { Metadados }\end{array}$ & \\
\hline $\begin{array}{l}\text { W3CProv }^{18} ; \text { PROV (PROV-DM, } \\
\text { PROV-wf Schema, ProvONE } \\
\text { Conceptual Model) } \\
\text { BBC Provenance Ontology }\end{array}$ & $\begin{array}{l}\text { Registro para } \\
\text { Proveniência }\end{array}$ & \\
\hline $\mathrm{CiTO}^{21} ; \mathrm{C}_{4} 0^{22} ; \mathrm{FOCO}^{23} ; \mathrm{C}_{2} \mathrm{~W}^{24}$ & Registro para Citação & \\
\hline
\end{tabular}




\begin{tabular}{|c|c|}
\hline $\begin{array}{l}\text { PRISM }^{25} \text {; BIBO; DEO } \\
\text { PSO }^{26} \text {; } \mathrm{PRO}^{29} \text {; } \mathrm{PWO}^{30} \text {; } \mathrm{SCORO}^{27} \text {; }\end{array}$ & $\begin{array}{l}\text { Registro para } \\
\text { Estrutura do } \\
\text { Documento }\end{array}$ \\
\hline PRISM; PSO; PRO; PWO; SCoRO & $\begin{array}{l}\text { Registro para Fase do } \\
\text { Ciclo de Vida do } \\
\text { Documento }\end{array}$ \\
\hline $\begin{array}{l}\text { Packages }^{32} \text { Researchobject.org } \\
\text { Packages }^{33} \text { Fairsharing.org }\end{array}$ & $\begin{array}{l}\text { Registro para Dados } \\
\text { de Pesquisa }\end{array}$ \\
\hline $\mathrm{EDM}^{34}$ & Registro de Obras \\
\hline \multicolumn{2}{|c|}{ Siglas/Acrônimos } \\
\hline $\begin{array}{l}{ }^{1} \text { Functional Requirements for Bibliographic } \\
\text { Records - entity-relationship model. } \\
2 \text { FRBR com Resource Description Framework. } \\
{ }^{3} \text { FRBR Object Oriented. } \\
{ }^{4} \text { FRBR in OWL2 DL Ontology. } \\
{ }^{5} \text { Bibliographic Framework as a Web of Data - } \\
\text { Linked Data Model and Supporting Services. } \\
6 \text { FRBR - aligned Bibliographic Ontology. } \\
7 \text { DataCite Metadata Schema. } \\
8 \text { Bibliographic Reference Ontology. } \\
{ }^{9} \text { Bibliographic Ontology. } \\
{ }^{10} \text { Metadata Encoding and Transmission } \\
\text { Standard. } \\
{ }_{11} \text { Metadata Object Description Schema. } \\
12 \text { Technical Metadata for Text. } \\
{ }^{13} \text { Encoded Archival Description. } \\
{ }^{14} \text { Library of Congress - description of works of } \\
\text { visual culture. } \\
{ }^{15} \text { Lightweight Information Describing Objects. } \\
{ }^{16} \text { Categories for the Description of Works of Art. } \\
{ }^{17} \text { Dublin Core Metadata. }\end{array}$ & $\begin{array}{l}{ }^{18} \text { Família W3CProv-Overview. } \\
{ }^{19} \text { W3CPROV Data Model e Workflows } \\
20 \text { Provenance Model. } \\
{ }^{21} \text { Citation Typing Ontology. } \\
{ }^{2} \text { Citation Counting and Context Characterization } \\
\text { Ontology. } \\
{ }^{23} \text { Functions of Citations Ontology. } \\
{ }^{2} \text { CiTO to Wordnet Ontology. } \\
{ }^{25} \text { Publishing Requirements for Industry } \\
\text { Standard Metadata. } \\
{ }^{26} \text { Discourse Elements Ontology. } \\
{ }^{27} \text { Documents Component Ontology. } \\
{ }^{28} \text { Publishing Status Ontology. } \\
{ }^{29} \text { Publishing Roles Ontology. } \\
{ }^{30} \text { Publishing Workflow Ontology. } \\
{ }^{31} \text { Scholarly Contributions and Roles Ontology. } \\
{ }^{32} \text { Activities of the Research Object community. } \\
\text { https://www.researchobject.org/. } \\
{ }^{33} \text { Registro de recursos Web/FAlR. } \\
\text { https://fairsharing.org. } \\
34 \text { Europeana Data Model. }\end{array}$ \\
\hline
\end{tabular}

Fonte: dados de pesquisa.

Os instrumentos presentes no Quadro 2 servem como referência para a avaliação da pertinência ou não do esforço de padronização, bem como para a análise de sua adequação e possível resposta aos requisitos do domínio. São instrumentos elaborados pela International Federation of Library Associations and Institutions (IFLA), International Council of Museums (ICOM) e International Council of Archives (ICA), os quais podem auxiliar na compreensão do domínio.

\section{Quadro 2 - Esforços para referência no domínio das GLAM}

\begin{tabular}{|l|l|}
\hline Instrumento & Escopo \\
\hline $\begin{array}{l}\text { IFLA/FRBR/LRM e IFLA/Standards/Conceptual } \\
\text { Models }\end{array}$ & Registro de obras bibliográficas \\
\hline ICOM/CIDOC-CRM & Registro de obras museológicas \\
\hline ICA/RiC-O e ICA/RiC-CM & Registro de obras arquivísticas \\
\hline
\end{tabular}


Fonte: dados de pesquisa.

Na busca de uma modelagem de referência, foi efetuada uma análise das propostas de representação (Quadro 1) em relação aos requisitos e pressupostos extraídos dos instrumentos preconizados pelo Quadro 2).

Um primeiro produto gerado e que se apresenta para debate foi elaborado no contexto das bibliotecas, trazendo, porém, a ótica de ativos de informação. Essa visão permite a existência de relações entre publicações científicas e dados de pesquisa, aproximando produtos documentais clássicos (relatórios de pesquisa, artigos e demais obras dessa categoria de publicações). Contudo, vale ressaltar que essa é uma versão preliminar do modelo que tem o objetivo de identificar os elementos principais relacionados a ativos de informação. As próximas versões deverão refinar e estender o modelo, com o possível incremento de conceitos e relações.

\section{Figura 1 - Proposta de modelo conceitual de referência incluindo a análise de cobertura dos modelos apresentados no Quadro 1.}

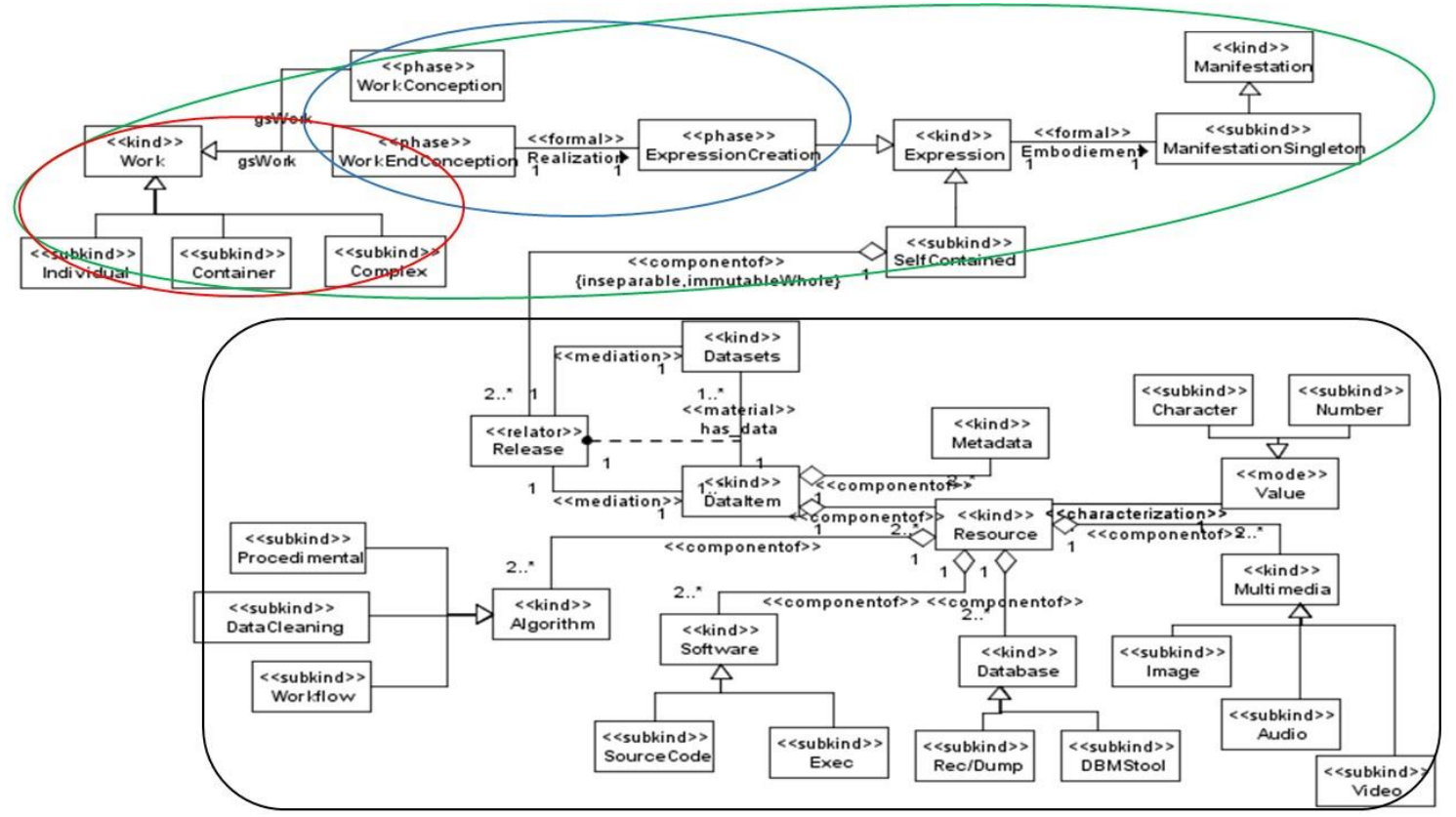

Fonte: os autores.

Em uma avaliação do escopo e da aplicação dos instrumentos identificados no Quadro 1, foram apontadas as áreas sinalizadas na Figura 1. Tais áreas apresentam o grau de sombreamento entre alguns modelos 
semânticos identificados, em especial os modelos que contemplam o uso de registros bibliográficos, metadados, proveniência e citação.

A aferição do escopo de Registro para Dados de Pesquisa pode suscitar a entrada de outros modelos semânticos, na medida em que houver evolução do modelo proposto na Figura 1 para abarcar outros domínios para além das GLAM. A análise de packages em Researchobject.org e Fairsharing.org também deve incrementar a coleção de modelos semânticos, possibilitando a busca da convergência nas representações e reduzindo a replicação de iniciativas.

\section{CONSIDERAÇÕES FINAIS}

Nesse relato, viu-se que o desenvolvimento de modelos incrementa proposta de simulações e reúso para atingirmos os resultados de investigações. Mondelli, Peterson e Gadelha Junior (2019, p. 9) convalidam essa percepção quando observam que: "We indicate, through a conceptual model, the essential aspects of the experiments that must be recorded and how they relate to different levels of reproducibility".

Complementarmente, percebe-se que no contexto da gestão dos Dados de Pesquisa o processo para adoção de registros sobre a semântica pode envolver a reunião de práticas ainda não tão amadurecidas pelos investigadores. Em estudo de caso sobre Research Data Management (RDM) na Princeton University, Li, Dressel e Hersey (2019) corroboram essa percepção quando observam que, apesar dos desafios que o tema impõe, muito ainda precisará ser incorporado no uso do RDM, em especial na gestão de metadados nas fases de preservação, análise, publicação e compartilhamento. Ainda na visão desses autores, o uso de estruturas de metadados associado com padrões e processos para representação pode enriquecer o RDM, além de viabilizar o processo de interoperabilidade entre domínios e plataformas distintas.

As iniciativas encaminhadas pela RDA (Research Data Alliance) têm procurado instigar os investigadores na direção do uso de requisitos e princípios FAIR. Contudo, a nossa percepção é que no domínio das GLAM ainda há espaço a ser preenchido por iniciativas de implementação, como o GO FAIR e 
convergência de modelos semânticos.

Os múltiplos formalismos semânticos identificados neste relato levam-nos a pressupor que a filosofia de reúso ainda precisa de amadurecimento, pois há diferentes modelos que podem convergir em suas definições. Existe um extenso campo para ser investigado com uma análise detalhada das sobreposições existentes nas áreas de cobertura e sombreamento observadas. Como elucidado na seção 3 a jornada é longa para a adoção de modelos semânticos bem fundamentados e convergentes. Pretende-se que este relato auxilie na redução do esforço para trilhar esse caminho.

\section{REFERÊNCIAS}

\section{ALLEMANG, D.; HENDLER, J. Semantic Web for the working ontologist:} Effective Modeling in RDFS e OWL. $2^{\text {nd }}$ edition. USA, Elsevier: Morgan Kaufmann, 2011.

ALMEIDA, F. A. S.; SILVA, A. M. Um modelo conceitual de gestão da informação em rede de cooperação de consórcio público intermunicipal: ferramentas de conformidade estatística e validação. Prisma.com (Portugal), n. 38, p. 3-18, 2019. Disponível em: http://hdl.handle.net/20.500.11959/brapci/112254. Acesso em: 24 jul. 2020.

ALMEIDA, J. P.; FALBO, R.; GUIZZARDI, G. Events as entities in ontologydriven conceptual modeling. Lecture Notes in Computer Science (including subseries Lecture Notes in Artificial Intelligence and Lecture Notes in Bioinformatics). Publisher: Springer, v. 11788 LNCS p. 469-483. 2019.

BARBA, L. Terminologies for Reproducible Research. December 2018. Disponível em: https://arxiv.org/pdf/1802.03311. Acesso em: 20 set. 2020.

BAKER, M. Is there a reproducibility crisis? Nature. v. 533, p. 452, 26 mai. 2016. Disponível em: https://www.nature.com/news/1-500-scientists-lift-the-lidon-reproducibility-1.19970. Acesso em: 20 set. 2020.

CARAN, G. M.; BIOLCHINI, J. C. A. Eixos conceituais do acesso à informação pelo deficiente visual: suportes cognitivo, social e tecnológico. Tendências da Pesquisa Brasileira em Ciência da Informação, v. 9, n. 2, 2016. Disponível em: http://hdl.handle.net/20.500.11959/brapci/119667. Acesso em: 25 jul. 2020.

CERRAO, N. G.; CASTRO, F. F. Perfil de aplicação de metadados baseado em FRBR e RDA. In: ENCONTRO NACIONAL DE PESQUISA EM CIÊNCIA DA INFORMAÇÃO (ENANCIB), 20. 2019. Anais [...] Florianópolis: ANCIB; UFSC, 
2019. Disponível em: http://hdl.handle.net/20.500.11959/brapci/122527. Acesso em: 25 jul. 2020.

CROFT, Willian. Typology and Universals. $2^{\text {nd }}$ edition. UK: Cambridge University Press, 2003.

FENLON, K. Modeling Digital Humanities Collections as Research Objects. ACM/IEEE Joint Conference on Digital Libraries 2019. Preprint of article published in Proceedings of ACM Conference JCDL '19. 2019. Disponível em: http://dx.doi.org/10.17613/jmns-kr78. Acesso em: 1 o fev. 2020.

FLORES, D.; PRADEBON, D. R. S.; CÉ, G. Análise do conhecimento teóricometodológico da preservação digital sob a ótica da OAIS, SAAI, ISO 14721 e NBR 15472. Brazilian Journal of Information Science, v. 11, n. 4, 2017. DOI: 10.5016/brajis.v11i4.7511. Acesso em: 25 jul. 2020.

GUIMARÃES, R. C. M.; ROGGE, M. B. Uma proposta de modelo conceitual para a construção de base de dados dos trabalhos de conclusão de curso. Informação@Profissões, v. 8, n. 1, p. 60-74, 2019. DOI: 10.5433/23174390.2019v8n1p60. Acesso em: 24 jul. 2020.

GUIZZARDI, G. Ontological Foundations for Structural Conceptual Models. Enschede, The Netherlands, CTIT PhD Thesis Series, No. 05-74 Telematica Instituut Fundamental Research Series, No. 015 (Tl/FRS/015). 2005. Disponível em: http://doc.utwente.nl/50826/1/thesis_Guizzardi.pdf. Acesso em: 10 set. 2020.

GUIZZARDI, G. On Ontology, ontologies, Conceptualizations, Modeling Languages, and (Meta)Models, Frontiers. In: VASILECAS, O.; EDLER, J.; CAPLINSKAS, A. (Eds.) Artificial Intelligence and Applications, Databases and Information Systems IV, ISBN 978-1-58603-640-8, IOS Press, Amsterdam. 2007

GUIZZARDI, G. Ontology, ontologies and the "I" of FAIR. Data Intelligence, v. 2, n.1, p. 181-191.2020. DOI: 10.1162/dint_a_00040.

HEILER, S. Semantic Interoperability. ACM Computing Surveys, v. 27, n.2, 1995

HENNING, P. C.; RIBEIRO, C. J. S.; SALLES, L. F.; MOREIRA, J. L. R.; SANTOS, L. O. B. S. Desmistificando os princípios FAIR: conceitos, métricas, tecnologias e aplicações inseridas no ecossistema dos dados FAIR. In: ENCONTRO NACIONAL DE PESQUISA EM CIÊNCIA DA INFORMAÇÃO (ENANCIB), 19. 2018. Anais [...] Londrina: ANCIB; UEL, 2018.

HENNING, P. C.; RIBEIRO, C. J. S.; SANTOS, L. O. B. S.; SANTOS, P. X. GO FAIR e os princípios FAIR: o que representam para a expansão dos dados de pesquisa no âmbito da Ciência Aberta. Em Questão, Porto Alegre, v. 25, n. 2, 
p. 389-412, maio/ago. 2019 DOI: http://dx.doi.org/10.19132/1808-5245252.389412.

INAZAWA, F. K.; BAPTISTA, S. G. Modelo conceitual de comunicação da informação para estudos de interação informacional baseado em competências conversacionais em serviço de referência. Perspectivas em Ciência da Informação, v. 17, n. 1, p. 169-184, 2012. Disponível em: http://hdl.handle.net/20.500.11959/brapci/36646. Acesso em: 24 jul. 2020.

IOANNIDIS, J. P. A. Why Most Published Research Findings Are False. PLOS Medicine. v.2, n.8, p. e124. 2005. https://doi.org/10.1371/journal.pmed.0020124

JAPIASSÚ, H.; MARCONDES, D. Dicionário básico de filosofia. 4a ed. Rio de Janeiro: Zahar, 2006.

JAPPUR, R. F.; FORCELLINI, F. A.; SPANHOL, F. J. Modelo conceitual para jogos educativos digitais. AtoZ: Novas Práticas em Informação e

Conhecimento, v. 3, n. 2, p. 116-127, 2014. DOI: 10.5380/atoz.v3i2.41344 Acesso em: 25 jul.2020.

KENT, W. Data and Reality. $1^{\text {st }}$ Books, 2000.

LAKATOS, E. M.; MARCONI, M. A. Metodologia Científica. 7ª . Edição. São Paulo: Atlas, 2017.

LATOUR, B. Ciência em Ação: como seguir cientistas e engenheiros sociedade afora. São Paulo: Editora Unesp, 2000.

LI, Y.; DRESSEL, W.; HERSEY, D. Research data management: What can librarians really help?. Grey Journal, v. 15, n. 1, p. 23-30. 2019.

MACHADO, L. M. O.; ALMEIDA, M. B.; SOUZA, R. R. What Researchers are Currently Saying about Ontologies: A Review of Recent Web of Science Articles. Knowledge Organization, v. 47. n. 3. 2020.

MARLOW, C. et al. Position paper, tagging, taxonomy, flickr, article, toread. [S. I.; s. n.], 2006. Disponível em:

http://www.danah.org/papers/WWW2006.pdf. Acesso em: 13 set. 2020.

MENDONÇA, A. V. M. O uso da análise do discurso do sujeito coletivo em Ciência da Informação. In: MULLER, S. P. M. Métodos para a pesquisa em ciência da informação. Brasília: Thesaurus. p. 149-170, 2007.

MONDELLI, M. L.; PETERSON, A. T.; GADELHA JUNIOR, L. M. R. Exploring Reproducibility and FAIR: Principles in Data Science Using Ecological Niche Modeling as a Case Study. In: GUIZZARDI, G; GAILLY, F.; MACIEL, S. P. Advances in Conceptual Modeling ER2019. Publisher: Springer International, 2019. 
MORAES, M. G.; SIMÕES, K. O. Controle terminológico da política de prevenção e controle do câncer: desafios da modelagem de políticas públicas. In: ENCONTRO NACIONAL DE PESQUISA EM CIÊNCIA DA INFORMAÇÃO (ENANCIB), 18., 2017. Anais [...] Marília: Ancib; Unesp, 2017. Disponível em: http://hdl.handle.net/20.500.11959/brapci/105046. Acesso em: 25 jul. 2020.

MOREIRA, W. Relações conceituais como elementos constitutivos essenciais dos sistemas de organização do conhecimento. Informação \& Informação, [S. I.], v. 24, n. 2, p. 1-30, nov. 2019. ISSN 1981-8920. Disponível em: http://www.uel.br/revistas/uel/index.php/informacao/article/view/37989. Acesso em: 16 dez. 2019. DOI: http://dx.doi.org/10.5433/1981-8920.2019v24n2p1.

MORENO, F. P. O modelo conceitual FRBR: discussões recentes e um olhar sobre as tarefas do usuário. Encontros Bibli: Revista Eletrônica de Biblioteconomia e Ciência da Informação, v. 14, n. 27, p. 47-68, 2009. DOI: 10.5007/1518-2924.2009v14n27p47. Acesso em: 25 jul. 2020.

MYLOPOULOS, J. Conceptual Modeling and Telos. 1992.

NATIONAL ACADEMIES OF SCIENCES, ENGINEERING, AND MEDICINE. Policy and Global Affairs. Reproducibility and Replicability in Science. Washington (DC): National Academies Press (US): May 7. 3, Understanding Reproducibility and Replicability. 2019. Disponível em:https:

//www.ncbi.nlm.nih.gov/books/NBK547546/ Acesso em: 20 set. 2020.

NOSEK, B. A.; SPIES, J. R.; MOTYL, M. Scientific Utopia: II. Restructuring Incentives and Practices to Promote Truth Over Publishability. Perspect Psychol Sci. v. 7, n. 6, p. 615-31. 2012 DOI: 10.1177/1745691612459058.

PADRON, M. F.; CRUZ, F. W.; SILVA, J. R. F. Modelos conceituais na ciência da informação: uma revisão de literatura. In: ENCONTRO NACIONAL DE PESQUISA EM CIÊNCIA DA INFORMAÇÃO (ENANCIB), 19. 2018. Anais [...] Londrina: ANCIB; UEL, 2018. Disponível em: http://hdl.handle.net/20.500.11959/brapci/102388. Acesso em: 24 de jul. de 2020

PAIVA, S. B. Modelo conceitual para o capital intelectual integrado à gestão do conhecimento. Informação \& Sociedade: Estudos, v. 22, n. Especial, 2012. Disponível em: http://hdl.handle.net/20.500.11959/brapci/93047. Acesso em: 25 jul. 2020.

RIBEIRO, C. J. S. Big Data: uma investigação com uso de dados abertos sobre acidentes de trabalho. In: ENCONTRO NACIONAL DE PESQUISA EM CIÊNCIA DA INFORMAÇÃO (ENANCIB), 15., 2014. Anais [...] Belo Horizonte, ANCIB; UFMG, 2014.

RIBEIRO, C. J. S.; PEREIRA, D. V. Em busca de uma interoperabilidade semântica na publicação de dados abertos governamentais: a experiência 
sobre os dados da Previdência Social. In: ENCONTRO NACIONAL DE PESQUISA EM CIÊNCIA DA INFORMAÇÃO (ENANCIB), 14., 2013. Anais [...] Florianópolis, ANCIB; UFSC, 2013.

ROBREDO, Jaime. Da ciência da informação revisitada aos sistemas humanos de informação. Brasília: Thesaurus Editora, 2003.

ROSS-HELLAUER, T.; FECHER, B.; SHEARER, K.; RODRIGUES, E. Pubfair: A Framework for Sustainable, Distributed. Open Science Publishing Services. 2019. Disponível em: https://www.researchgate.net/publication/335600629_Pubfair_A_Framework_fo r_Sustainable_Distributed_Open_Science_Publishing_Services. Acesso em: 20 jul. 2020.

SANDVE, G. K.; NEKRUTENKO, A.; TAYLOR, J.; HOVIG, E. Ten Simple Rules for Reproducible Computational Research. PLoS Comput. Biol. 9, e 1003285. 2013.

SILVA, J. H.; SIEBRA, S. A.; ALBUQUERQUE, M. E. B. C. Apontamentos sobre a representação da informação no modelo conceitual OAIS. Encontro Nacional de Pesquisa em Ciência da Informação (XIX Enancib), 2018. Disponível em: http://hdl.handle.net/20.500.11959/brapci/103752. Acesso em: 24 jul. 2020.

SIMIONATO, A. C.; ARAKAKI, F. A.; SANTOS, P. L. V. A. C. Descrição em bibliotecas, arquivos, museus e galerias de arte: linkando recursos e comunidades. Informação \& Informação, v. 22, n. 2, 2017. 10.5433/19818920.2017v22n2p449. DOI:10.5433/1981-8920.2017v22n2p449. Disponível em: http://www.brapci.inf.br/v/a/28926. Acesso em: 10 jun. 2020.

SIMIONATO, A. C.; SANTOS, P. L. V. A. C. Modelo conceitual dilam: integração entre arquivos, bibliotecas e museus. Informação \& Sociedade: Estudos, v. 27, n. 2, 2017. DOI: 10.22478/ufpb.18094783.2017v27n2.30902. Acesso em: 30 jul. 2020

SOWA, J. F. Knowledge Representation. Ed. CAEd. 2000.

STODDEN, V. Reproducing Statistical Results. Annual Review of Statistics and Its Application, v. 2, n. 1, p. 1-19, 2015.

VERDONCK, M.; GAILLY, F.; PERGL, R.; GUIZZARDI, G.; MARTINS, B.; PASTOR, O. Comparing traditional conceptual modeling with ontology-driven conceptual modeling: An empirical study. Information Systems, v. 81, p. 92103, 2019.

WEBER, B. Next Generation Modeling Environments. In: LAENDER, A. H. F.; PERNICI, B.; LIM, E-P.; OLIVEIRA, J. P. M. (Eds.). Conceptual Modeling.

Proceedings of 38th International Conference. ER 2019, Salvador, Brazil, November 4-7, 2019. 
WIKIPEDIA. Position Paper. 2020. Disponível em https://en.wikipedia.org/wiki/Position_paper. Acesso em: 10 set. 2020.

WILKINSON, M.; DUMONTIER, M.; AALBERSBERG, I. et al. The FAIR Guiding Principles for scientific data management and stewardship. Scientific Data, 160018, 3, 2016.

WILKINSON, M. D.; SANSONE, S.-A.; SCHULTES, E.; DOORN, P.; SANTOS, L. O. B. S.; DUMONTIER, M. A design framework and exemplar metrics for FAIRness. Sci. Data, 5:180118. 2018. DOI:10.1038/sdata.2018.118.

ZAHER, C. R.; GOMES, H. E. Da bibliografia à ciência da informação: um histórico e uma posição. Ciência da Informação, v. 1, n. 1, 11. 1972.

ZINS, C.; DEBONS, A.; BEGHTOL, C.; et al. Mapa do conhecimento da ciência da informação: implicações para o futuro da área. Brazilian Journal of Information Science, v. 1, n. 1, 2007. DOI: 10.5016/brajis.v1i1.32. Acesso em: 13 set. 2020.

\title{
FAIR PRINCIPLES AND INTEROPERABILITY: REPRODUCIBILITY WITH ONTOLOGY-DRIVEN CONCEPTUAL MODELLING
}

\begin{abstract}
Introduction: conceptual models can be used as reference artifacts to help researchers understand some knowledge domains. These artifacts help us minimize the semantic distance between how we represent and interpret information. Taking into account that data should be shared among Science and Technology institutions, as well as the reproducibility crisis, this report aims at investigating existing models and formalisms to represent in GLAM (Galleries, Libraries, Archives and Museums). Objective: our intent is to establish a list of standards used such as vocabularies, conceptual models and ontologies toward to convergence of representation research information assets. Methodology: blending methods and epistemological approaches, this position paper has collected some standards for information representation in order to align these artifacts so that they can better represent information assets. Results: this paper presents a set of identified instruments thar are also categorized according to their applicability, by using a semantic model approach. Conclusions: this report focuses on aligning the standards in order to reduce them and make them work together with conceptual models in order to allow interoperability and use of FAIR principles.
\end{abstract}

Descriptors: Reproducibility. FAIR. Interoperability. Conceptual Model. Representation.

\section{PRINCIPIOS FAIR E INTEROPERABILIDAD: REPRODUCIBILIDAD CON EL USO DE MODELADO CONCEPTUAL ORIENTADO A ONTOLOGÍAS}




\section{RESUMEN}

Introducción: El desarrollo de modelos conceptuales como artefactos de referencia hacia la comprensión de campos del conocimiento contribuye auxiliando a reducir la distancia semántica entre la representación y la interpretación de las informaciones. Basándose en el contexto del uso compartido de los datos en los esfuerzos por el acceso abierto a la producción en Ciencia y Tecnología, así como las dificultades ocasionadas por la crisis de la reproducibilidad, este relato analiza la existencia de ejemplos semánticos que representen el contexto GLAM (Galleries, Libraries and Archives). Objetivo: Propone también el análisis de esta lista de modelos en búsqueda de homogeneizar las representaciones de los objetos componentes de las investigaciones, teniendo en cuenta los principios FAIR, y de establecer una referencia para permitir la convergencia en las representaciones. Metodología: Este position paper combina métodos y enfoques epistemológicos y utiliza la investigación exploratoria con el análisis bibliográfico y documental para identificar patrones de representación. Resultados: Presenta el conjunto de instrumentos que viabilicen el enriquecimiento semántico, identificados en la revisión de la literatura, además de una propuesta de modelo conceptual que contempla publicaciones y una tipología de datos de investigación. Conclusiones Este relato señala la necesidad de converger patrones de representación conjuntamente con modelos conceptuales, como una manera de hacer viables la interoperabilidad y el uso de principios FAIR.

Descriptores: Reproducibilidad. FAIR. Interoperabilidad. Modelado Conceptual. Representación.

Recebido em: 15/09/2020

Aceito em: 30/09/2020 